\title{
FAILURE OF EQUIVALENCE OF DIMENSION CONCEPTS FOR METRIC SPACES
}

\author{
BY PRABIR ROY ${ }^{1}$ \\ Communicated by Deane Montgomery, May 7, 1961
}

Introduction. The three classical set-theoretic concepts of dimensions for topological spaces are [2, p. 153]: small inductive dimension -denoted by ind-such that ind $(S)=-1$ if $S$ is empty, $\operatorname{ind}(S) \leqq n$ if for every point $p \in S$ and open set $U$ containing $p$ there is an open set $V$ with $p \in V \subset U$ and ind(bdry $V) \leqq n-1$, and ind $(S)=n$ if ind $(S) \leqq n$ but ind $(S) \leqq n-1$ is not true; large inductive dimension-denoted by Ind-such that $\operatorname{Ind}(S)=-1$ if $S$ is empty, $\operatorname{Ind}(S) \leqq n$ if for every closed set $C$ and open set $U$ containing $C$ there is an open set $V$ with $C \subset V \subset U$ and $\operatorname{Ind}($ bdry $V) \leqq n-1$, and $\operatorname{Ind}(S)=n$ if $\operatorname{Ind}(S) \leqq n$ but $\operatorname{Ind}(S) \leqq n-1$ is not true; and covering dimension-denoted by $\operatorname{dim}$-such that $\operatorname{dim}(S)=-1$ if $S$ is empty, $\operatorname{dim}(S) \leqq n$ if every open cover of $S$ has an open refinement of order $\leqq n+1-$ no point of $S$ belongs to more than $n+1$ many members of the refinement, and $\operatorname{dim}(S)=n$ if $\operatorname{dim}(S) \leqq n$ but $\operatorname{dim}(S) \leqq n-1$ is not true. It is well known [2, p. 153] that for separable metric spaces $S$, ind $(S)=\operatorname{Ind}(S)$ $=\operatorname{dim}(S)$. For arbitrary metric spaces $S$, only recently Katětov [3] showed that $\operatorname{Ind}(S)=\operatorname{dim}(S)$. However, the question $[1$, p. 3]:

$$
\text { is } \operatorname{ind}(S)=\operatorname{dim}(S) \text { for arbitrary metric spaces } S \text { ? }
$$

remained open. We answer this question in the negative by demonstrating the following assertion.

TheOREM. There is a complete metric space $\Delta$ such that $\operatorname{ind}(\Delta)=0$ but $\operatorname{dim}(\Delta)=1$.

We shall give here only a description of the space $\Delta$, accompanied with remarks intended to aid in visualizing the space, and some idea of the proof. The details of the proof will be published elsewhere.

Notation. The symbol $\varnothing$ will denote the empty set. By a sequence we shall mean a function defined on either the set of non-negative integers, or the set of positive integers, or any initial segment of either of them. With this in mind the following notations are adopted. $X$ $=$ the set of all finite sequences of real numbers defined on initial

${ }^{1}$ Supported by National Science Foundation Grant (NSF-G21514). The author is deeply grateful to his teachers: F. B. Jones and J. S. Mac Nerney, and to I. Glicksberg and D. Montgomery for their interest and encouragement and to L. F. McAuley for communicating the problem to the author. 
segments of the non-negative integers such that if $x \in X$ then $x(i)=0$ only in case $i=0$. Furthermore if $x \in X$ then $|x|$ will denote the greatest integer for which $x$ is defined. $Y=$ the set of all reversible (=oneto-one) sequences of positive numbers defined on the set of all positive integers. $Z=$ the set of all infinite sequences of positive numbers defined on the set of all positive integers. If $r$ is a positive number then $Y_{r}$ will denote the set of all members of $Y$ which take on the value $r$, and $F_{r}$ will denote a reversible function from the positive numbers onto $Y_{r}$.

Points of $\Delta$. There are two types of points of $\Delta$ and they will be denoted by $P_{1}$ and $P_{2}$.

$P_{1}=$ the set of all infinite sequences of nonzero real numbers defined on the set of all positive integers.

Remark. One may visualize $P_{1}$ by imagining two rows of balls from some power-of-the-continuum dimensional complete Hilbert space, each row having as many balls as there are real numbers, with the top row being matched up with the set of positive numbers and the bottom row being matched up in a natural fashion with the negative numbers. Now imagine that inside each of the balls we have again two rows of balls as originally and so on (countably many times). Thus for each point in $P_{1}$ one can choose a systematic nested sequence of balls and conversely.

$P_{2}=X \times Y \times Z$, with $p_{X}, p_{Y}$, and $p_{z}$ denoting the coordinates of a point $p \in P_{2}$.

REMARK. The points in $P_{2}$ are best visualized in pairwise disjoint sets of the form $S_{(x, y)}=\left\{p \in P_{2} \mid p_{X}=x\right.$ and $\left.p_{Y}=y\right\}$ with $x \in X$ and $y \in Y$. Given an $x \in X$ and $y \in Y$ and viewing the whole space $\Delta$ as a ball we have that $x$ picks out an unique ball $B$. In the two rows of next smaller sized balls inside $B, y$ picks out a sequence of balls from the top rows and the corresponding sequence from the bottom row. Imagine that $S_{(x, y)}$ is located inside $B$ and at the "end" of the two parallel sequences of balls indicated by $y$. Now since the location with regard to $S_{(x, y)}$ of a point $p \in S_{(x, y)}$ is dependent only on $p_{z}$ (an infinite sequence of positive numbers), we can view $S_{(x, y)}$ itself as a "cantor" type set obtained by taking a row of power-of-the-continuum many balls (matched up with the positive numbers) and a similar row of smaller balls inside each of previous balls and so on (countably many times).

Regions of $\Delta$. By regions we mean what is often called a topological basis. That the sets described below indeed form a topological basis is not proved here. There are two types of regions and they are denoted by $\Gamma_{1}$ and $\Gamma_{2}$. 
$R$ is a member of $\Gamma_{1}$ only in case there is an $x \in X$ such that $R=R^{1} \cup R^{2}$, where

$$
R^{1}=\left\{p \in P_{1} \mid p(i)=x(i) \text { for } i=1, \cdots,|x|, \text { if }|x|>0\right\}
$$

and

$$
R^{2}=\left\{p \in P_{2}|| p_{x}|\geqq| x \mid ; p_{X}(i)=x(i) \text { for } i=1, \cdots,|x| \text {, if }|x|>0\right\} \text {. }
$$

Such a region $R$ will be denoted by $R_{x}$.

REMARK. The members of $\Gamma_{1}$ can be thought of as dilapidated versions of the balls involved in the description of $P_{1}$-each modified ball consists of the points of $P_{1}$ that are left in the ball and the points of $P_{2}$ that are placed in the ball.

$R$ is a member of $\Gamma_{2}$ only in case there is a positive integer $n$ and a point $p \in P_{2}$ such that $R=R^{0} \cup R^{+} \cup R^{-}$, where

(1) $R^{0}=\left\{q \in P_{2} \mid q_{X}=p_{X} ; q_{Y}=p_{Y} ; q_{Z}(i)=p_{Z}(i)\right.$

$$
\text { for } i=1, \cdots, n-1 \text {, if } n>1\} \text {, }
$$

$$
R^{+}=\bigcup_{i=1}^{\infty} R_{\gamma(p, n,+)_{i}} \text { and } R^{-}=\bigcup_{i=1}^{\infty} R_{\gamma(p, n,-)_{i}}
$$

where $\left\{\gamma(p, n,+)_{i}\right\}_{1}^{\infty}$ and $\left\{\gamma(p, n,-)_{i}\right\}_{1}^{\infty}$ are two infinite reversible sequences of members of $X$ such that if $j$ is a positive integer, then

$$
\begin{aligned}
\left|\gamma(p, n, \pm)_{j}\right| & =\left|p_{X}\right|+n+1, \\
\gamma(p, n, \pm)_{j}(i) & =p_{X}(i) \quad \text { for } i=0, \cdots,\left|p_{X}\right|, \\
\gamma(p, n, \pm)_{j}\left(\left|p_{X}\right|+1\right) & = \pm p_{Y}(n+j-1), \\
\gamma(p, n, \pm)_{j}\left(\left|p_{X}\right|+2\right) & =\mp F_{p_{Y}(n+j-1)}{ }^{-1}\left(p_{Y}\right), \text { and if } n>1 \\
\gamma(p, n, \pm)_{j}\left(\left|p_{X}\right|+2+i\right) & =\mp p_{Z}(i) \quad \text { for } i=1, \cdots, n-1 .
\end{aligned}
$$

Such a region $R$ will be denoted by $R_{(p, n)}$.

REMARK. The members of $\Gamma_{2}$ are the crucial items in the space $\Delta$ for they hold together the hitherto highly scattered space to the precise extent necessary to produce the peculiar disparity of dimensions. In order to picture a member $R_{(p, n)}$ of $\Gamma_{2}$, since it is a neighborhood of $p \in P_{2}$, recall the definition of $P_{2}$ and the remark following it. Conditions (1) and (4) state that $R_{(p, n)}$ lies inside that ball $B$ designated by $p_{x}$. The part of $S_{\left(p_{X}, p_{Y}\right)}$ belonging to $R_{(p, n)}$ is determined by (1). The rest of $R_{(p, n)}$ consists of two sequences of balls (as indicated by (2)) of which the sequence $\left\{R_{\gamma(p, n,+)_{j}}\right\}_{1}^{\infty}$ is obtained as follows: to begin with, as indicated by (5), discard the first $n-1$ balls in the top sequence of balls determined by $p_{Y}$; secondly, inside each of the remaining balls $B^{\prime}$, select a next sized ball from the bottom row as specified by (6) 
(note that the latest ball obtained will not be selected at this stage for any other $R_{\left(p^{\prime}, n^{\prime}\right)}$ with $\left.p^{\prime} \notin S_{\left(p_{X}, p_{Y}\right)}\right)$ and continue the process $n-1$ many more times, always selecting a ball from the bottom row of the previous ball as stated precisely in (7), until a small ball is determined, whose size is given by (3) and whose position in the structure of "bottom rows of bottom rows" of balls inside $B^{\prime}$ is the same as the position of the ball corresponding to $R^{0}$ is to the structure of balls involved in the "cantor" type generation of $S_{\left(p_{X}, p_{Y}\right)}$; finally, the region in $\Gamma_{1}$ corresponding to this latest ball obtained will be a term in the sequence of balls we are describing. The sequence $\left\{R_{\gamma(p, n,-)_{j}}\right\}_{1}^{\infty}$ is obtained by the "mirror image" of the above process-of course applied to the bottom sequence of balls determined by $p_{Y}$, as is specified in the change of signs in (6) and (7).

A sequence of open coverings of $\Delta$. For each positive integer $n$ let

$$
\begin{aligned}
G_{n}= & \left\{R\left|R=R_{x} \in \Gamma_{1} ;\right| x \mid \geqq n\right\} \\
& \cup\left\{R\left|R=R_{(q, m)} \in \Gamma_{2} ;\right| q_{X} \mid+m \geqq n\right\} .
\end{aligned}
$$

That $\left\{G_{n}\right\}_{1}^{\infty}$ is a decreasing sequence of open covers of $\Delta$ follows directly from the definition.

The space $\Delta$ is metrizable. To prove this we use the above sequence of coverings in applying the following theorem:

Moore's metrization theorem [4, pp. 18, 19, 21]. A topological space $S$ is metrizable if

(i) $S$ is a Hausdorff space, and

(ii) there is decreasing sequence $\left\{H_{1} \supset H_{2} \supset H_{3} \supset \ldots\right\}$ of open coverings of $S$ such that for every point $p$ and every open set $U$ containing $p$ there is a positive integer $N$ with the property that if $h_{1}$ and $h_{2}$ belong to $H_{N}, p \in h_{1}$, and $h_{1} \cap h_{2} \neq \varnothing$, then $h_{1} \cup h_{2} \subset U$.

The space $\Delta$ is complete. In fact the space $\Delta$ is complete with respect to the sequence $\left\{G_{n}\right\}_{1}^{\infty}$ of open coverings-that is, if $\left\{C_{n}\right\}_{1}^{\infty}$ is a sequence of nonvoid closed sets and $\left\{g_{n}\right\}_{1}^{\infty}$ is a sequence of regions such that for each positive integer $n, C_{n+1} \subset C_{n} \subset g_{n} \in G_{n}$, then $\bigcap_{n=1}^{\infty} C_{n} \neq \varnothing$.

$\operatorname{ind}(\Delta)=0$. This follows from the fact that none of the regionswhich form a topological basis for $\Delta$-has a boundary.

$\operatorname{dim}(\Delta)>0$. To prove this in view of Katětov's result, it is enough to show that $\operatorname{Ind}(\Delta)>0$. In particular we show that the space $\Delta$ is not the union of two disjoint open sets $U^{+}$and $U^{-}$containing respec- 
tively the disjoint closed sets $C^{+}=\left\{p \in P_{1} \mid p(i)>0\right.$ for $\left.i=1,2,3, \cdots\right\}$ and $C^{-}=\left\{p \in P_{1} \mid p(i)<0\right.$ for $\left.i=1,2,3, \cdots\right\}$, and this may be done by using the completeness of $\Delta$ in conjunction with the following observation:

LEMмA. If $x \in X$ and $\Delta$ is the union of two open sets $U^{+}$and $U^{-}$which have on the region $R_{x}$ the following effect:

$$
U^{+} \supset K^{+} \text {and } U^{-} \supset K^{-}
$$

where $K^{+}$and $K^{-}$are disjoint closed sets contained in $P_{1} \cap R_{x}$ with the property that if $p \in K^{+}$(respectively $K^{-}$) and $n$ is a positive integer then there is an infinite set $T$ of positive numbers such that for each number $t \in T$ there is a point $q \in K^{+}\left(\right.$resp. $\left.K^{-}\right)$with $q(|x|+i)=p(|x|+i)$ for $i=1, \cdots, n-1$ if $n>1$, and $q(|x|+n)=t$ (resp. $-t)$; then $U^{+}$and $U$ - have the same effect on $R_{x^{\prime}}$ for some $x^{\prime} \in X$ with $\left|x^{\prime}\right|=|x|+1$, and $x^{\prime}(i)=x(i)$ for $i=0, \cdots,|x|$.

REMARK. In other words if $U^{+}$and $U^{-}$contain respectively a closed set $K^{+}$at the very top of $R_{x}$ and a closed set $K^{-}$at the very bottom of $R_{x}$ where each of $K^{+}$and $K^{-}$is generated by "cantor" type process of taking "infinitely many balls inside each previous ball" (sticking with top rows for $K^{+}$and bottom rows for $K^{-}$), then $U^{+}$and $U^{-}$do the same for some next sized region $R_{x^{\prime}}$ inside $R_{x}$. Note that $K^{-}$is not required to be the "mirror" image of $K^{+}$nor is it claimed that the appropriate closed sets in $R_{x^{\prime}}$ are smaller copies of $K^{+}$and $K^{-}$.

$\operatorname{dim}(\Delta) \leqq 1$. This can be proved in a straightforward manner: given an open cover of $\Delta$, we can get a refinement of order $\leqq 2$ consisting of regions.

\section{REFERENCES}

1. P. S. Aleksandrov, The present status of the theory of dimension, Uspehi Mat. Nauk (N.S.) (6) 45 (1951), 43-68. (Russian) =Amer. Math. Soc. Transl. (2) 1 (1955), $1-26$.

2. W. Hurewicz and H. Wallman, Dimension theory, Princeton Math. Series no. 4, 1948.

3. M. Katětov, On the dimension of metric spaces, Dokl. Akad. Nauk SSSR (N.S.) 79 (1951), 189-191. (Russian) 13-28.

4. R. L. Moore, A set of axioms for plane analysis situs, Fund. Math. 25 (1935),

\section{University of North Carolina and} INSTITUTE FOR ADVANCED STUdy 